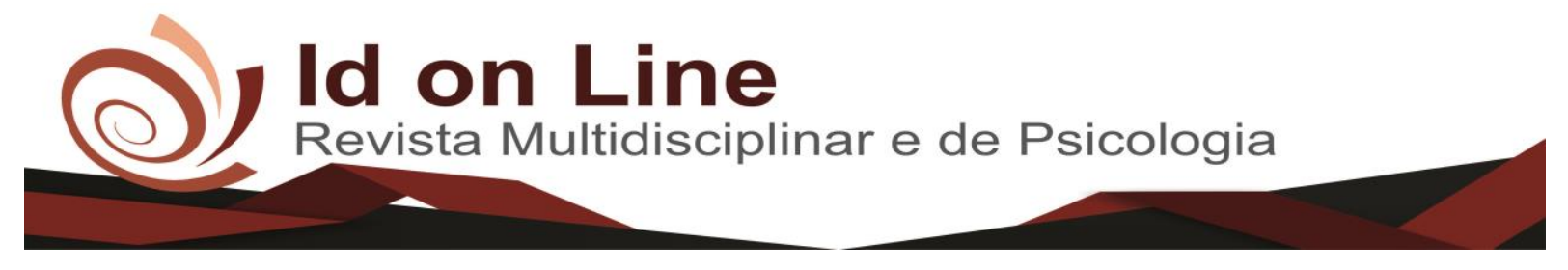

DOI: 10.14295/idonline.v15i55.3030

Artigo de Revisão

\title{
Comunicação efetiva da equipe multiprofissional na promoção da segurança do paciente em ambiente hospitalar
}

\author{
Tatiane de Oliveira Santos ${ }^{1}$, Maria Adriely Cunha Lima ${ }^{4}$, Victória Santos Alves ${ }^{2}$, \\ Maria Caroline Andrade Ribeiro ${ }^{2}$, Raquel Santos Alves ${ }^{3}$, Mércia Rocha Souza ${ }^{3}$, \\ Fernanda Vasconcelos Prado Correia ${ }^{4}$, Ana Carolina Amorim Oliveira ${ }^{4}$, \\ Larissa Ferreira Sales ${ }^{2}$, Halley Ferraro Oliveira ${ }^{5}$
}

\begin{abstract}
Resumo: Demonstrar a importância de uma boa comunicação efetiva entre a equipe multiprofissional para a segurança do paciente. Trata-se de uma revisão integrativa de artigos encontrados através de descritores das bases de dados BVS e SciELO, em que foi encontrado 15 artigos após a seleção baseada nos critérios de inclusão e de exclusão. Observou-se, que os artigos descrevem resultados similares, visto que explicam a necessidade e a relevância da comunicação efetiva não só na qualidade do atendimento, mas também na segurança do paciente durante a assistência multiprofissional. É valido mencionar, que a comunicação efetiva não somente interfere no desfecho clínico do paciente, mas também promove bem-estar dos profissionais, dado que há redução do estresse entre os membros, por exemplo. A efetiva comunicação da equipe gera benefícios mútuos, através da melhora na assistência e na segurança, e para os profissionais, uma vez que melhora a qualidade do ambiente no trabalho.
\end{abstract}

Palavras-chave: Comunicação. Equipe de assistência ao paciente. Segurança do paciente.

\section{Effective communication by the multidisciplinary team in promoting patient safety in the hospital environment}

\begin{abstract}
Demonstrate the importance of good effective communication between the multidisciplinary team for patient safety. It is an integrative review of articles found through descriptors from the VHL and SciELO databases, in which 15 articles were found after the selection based on the inclusion and exclusion criteria. It was observed that the articles describe similar results, since they explain the need and the relevance of effective communication not only in the quality of care, but also in patient safety during multiprofessional care. It is worth mentioning that effective communication not only interferes with the patient's clinical outcome, but also promotes the well-being of professionals, given that there is a reduction in stress between members, for example. The effective communication of the team generates mutual benefits, through the improvement in assistance and safety, and for the professionals, since it improves the quality of the environment at work.
\end{abstract}

Keywords: Communication. Patient care team. Patient safety.

\footnotetext{
${ }^{1}$ Enfermeira graduada pela Universidade Tiradentes (UNIT), mestranda do Programa de Pós-Graduação em Ciências Fisiológicas da Universidade Federal de Sergipe (UFS), Aracaju-SE, Brasil. enfermeiraconectada@gmail.com;

${ }^{2}$ Acadêmicos (as) do Curso de Enfermagem da Universidade Tiradentes (UNIT), Aracaju- SE, Brasil. victorialvesantos@outlook.com;

${ }^{3}$ Acadêmicas do Curso de Enfermagem do Centro Universitário Maurício de Nassau, Aracaju- SE, Brasil. victorialvesantos@outlook.com

${ }^{4}$ Acadêmicas do Curso de Medicina da Universidade Tiradentes (UNIT), Aracaju- SE, Brasil. mariaadrielycunha@hotmail.com;

${ }^{5}$ Médico, docente adjunto do Departamento de Medicina da Universidade Federal de Sergipe (UFS) e Universidade Tiradentes (UNIT), Aracaju- SE, Brasil. halleyoliveira62@gmail.com.
} 


\section{Introdução}

A comunicação efetiva e o trabalho da equipe multiprofissional na saúde são compreendidos como determinantes da qualidade e segurança do paciente. Sendo assim, a segurança do cliente constitui um dos grandes desafios dos cuidados de saúde nos dias atuais e, por isso, objetiva-se nas instituições de saúde, incluindo o hospital, diminuir danos e riscos, assegurar o bem-estar do paciente, que, quando alcançado, pode também diminuir o tempo de internação e tratamento. Nesse sentido, entre o conjunto de medidas para a garantia de uma assistência segura estão o seguimento de protocolos e a comunicação clara e objetiva entre os profissionais da saúde, de modo a minimizar erros nos cuidados. (OLINO et al., 2019; SANTOS et al., 2017)

A comunicação efetiva, seja ela verbal e/ou não verbal, constitui-se como uma das metas internacionais mais importantes para prevenir falhas ou eventos adversos evitáveis, sendo também um instrumento terapêutico fundamental no cuidado ao paciente (SANTOS et al., 2017). Essa comunicação, segundo Agência Nacional de Vigilância Sanitária (ANVISA) deve envolver uma linguagem clara, técnicas de comunicação, em vista de promover uma cultura de segurança nas instituições de saúde (BIASIBETTI et al., 2019).

As falhas na comunicação entre os profissionais de saúde, bem como entre esses e os pacientes ou acompanhantes, têm sido um dos principais fatores que contribuem para a ocorrência de eventos adversos (conjunto de falhas na segurança do paciente: organizacionais, práticas ou comportamentos profissionais inadequados decorrentes de diversos fatores) e, consequentemente, para a diminuição da referida segurança (SOUZA et al., 2014; SIMAN et al., 2017).

Segundo os dados do Instituto Brasileiro para Segurança do Paciente (2017), nota-se que a comunicação ineficaz está entre as causas de $70 \%$ dos erros cometidos na atenção à saúde. Além disso, um estudo realizado em um hospital evidenciou que quase $75 \%$ dos profissionais da saúde legitimaram que não há cooperação entre as equipes. Esse alto índice de erros na assistência à saúde levou o Ministério da Saúde juntamente com a Anvisa a instituir o Programa Nacional de Segurança do Paciente em 2013 com o objetivo de prevenir e reduzir a ocorrência de incidentes, qualificar o cuidado, onde a união do trabalho em equipe e a comunicação fazem parte desse conjunto de metas (BOHRER et al., 2016; BRASIL, 2013).

Desse modo, a segurança do paciente é primordial para a boa formação de profissionais de saúde, principalmente mediante a melhoria que pode proporcionar na forma de prestação de 
serviços aos usuários pelas instituições, com a amplificação de conhecimentos e métodos científicos, a fim de minimizar a incidência e impactos dos danos e aumentar a recuperação com qualidade para alcançar um sistema de saúde confiável (PEREIRA et al., 2015).

Neste sentido, a justificativa desse artigo está pautada na importância da comunicação efetiva da equipe multidisciplinar na promoção da segurança ao paciente em ambiente hospitalar. Portanto, o presente estudo tem como objetivo demonstrar a importância de comunicação eficiente da equipe multiprofissional para a promoção de segurança ao paciente em ambiente hospitalar.

\section{Metodologia}

Trata-se de uma revisão integrativa, com uma abordagem qualitativa, estruturada mediante as seguintes etapas: reflexão e construção da pergunta norteadora; seleção e busca de artigos; significação das informações a serem retiradas dos estudos selecionados; avaliação crítica dos trabalhos selecionados; discussão dos resultados e apresentação da revisão integrativa. Para a primeira etapa, as seguintes perguntas norteadoras foram elaboradas: "A equipe multiprofissional de saúde realiza a comunicação efetiva e qualificada nas unidades? A equipe está qualificada e preparada para realizar a melhor comunicação em prol da segurança do paciente?".

Foi realizada a busca dos artigos, baseada na análise dos dados disponibilizados na Biblioteca Virtual em Saúde (BVS), Scientific Electronic Library Online (SciELO), foram utilizadas. Os descritores "Comunicação", "Segurança do Paciente" e "Equipe de Assistência ao Paciente " foram utilizados de acordo com a classificação dos Descritores em Ciências da Saúde (DECS) e combinados com o auxílio do operador booleano AND. Primeiramente utilizou-se "Comunicação" AND “Equipe de Assistência ao Paciente”, em seguida, "Segurança do Paciente" AND “Comunicação" AND "Equipe de Assistência ao Paciente", quando combinados forneceram um quantitativo de 307 artigos.

Mediante a aplicação dos critérios de inclusão: textos completos, disponíveis online, nos idiomas português, inglês e espanhol, publicados entre 2015 a 2019; e de exclusão: artigos que não apresentavam significativa relação com a temática abordada e estudos de domínio privado ou indisponíveis para download, um total de 227 (BVS) e 56 (SciELO) artigos foram encontrados nas bases. O recorte temporal foi baseado nos últimos cinco anos. 
Ao aplicar os critérios de elegibilidade, apenas 15 artigos foram selecionados no total, pois abordaram de maneira satisfatória a troca de comunicação da equipe multiprofissional na assistência e segurança dos pacientes. Por conseguinte, os artigos selecionados foram submetidos à leitura prévia dos títulos e resumos, para melhor compreensão e identificação dos objetivos dos estudos e dos métodos utilizados. No segundo momento de leitura, por sua vez, foi realizada uma análise mais aprofundada, buscando identificar aspectos ligados intimamente com a temática abordada.

Trata-se de uma pesquisa bibliográfica, onde os dados obtidos seguiram princípios éticos, segundo a Lei dos Direitos Autorais número 12.853/2013. As informações contidas neste estudo foram citadas de maneira fidedigna, conforme bibliografia selecionada e preconizada pela Associação Brasileira de Normas Técnicas (ABNT) NBR 6023 e NBR 10520.

\section{Resultados}

O quadro 1 apresenta a caracterização dos artigos analisados a partir da descrição do autor, ano de publicação, título e as principais considerações de cada estudo observado. Verifica-se que dos 10 artigos encontrados, as sínteses alinham-se entre si, entrando em consenso sobre as opiniões quanto à importância da comunicação na qualidade do atendimento e da segurança do paciente na assistência da equipe multiprofissional. Além disso, foi possível identificar novas técnicas e ferramentas que auxiliam na comunicação entre as equipes, consumando assim as formas de disseminar as informações importantes de cada cliente.

Quadro 1. Artigos selecionados e suas características.

\begin{tabular}{|c|c|c|}
\hline Autores & Título & Síntese/considerações \\
\hline $\begin{array}{l}\text { CHILDRESS, } \\
2015 .\end{array}$ & $\begin{array}{l}\text { Comunicação da equipe: trata-se de } \\
\text { segurança do paciente. }\end{array}$ & $\begin{array}{l}\text { A comunicação dos profissionais afeta tanto na } \\
\text { qualidade do cuidado ao paciente, quanto na } \\
\text { satisfação da equipe. }\end{array}$ \\
\hline $\begin{array}{l}\text { NOGUEIRA, } \\
\text { RODRIGUES, } \\
2015 .\end{array}$ & $\begin{array}{l}\text { Comunicação Efetiva no Trabalho em } \\
\text { Equipe em Saúde: Desafio Para a } \\
\text { Segurança do Paciente. }\end{array}$ & $\begin{array}{l}\text { Comunicação deficiente reflete em uma } \\
\text { assistência insegura para o paciente, } \\
\text { contribuindo consecutivamente para, na maioria } \\
\text { das vezes, um desfecho desfavorável dos casos. }\end{array}$ \\
\hline
\end{tabular}




\begin{tabular}{|c|c|c|}
\hline $\begin{array}{l}\text { FASSARELLA et } \\
\text { al., } 2017 .\end{array}$ & $\begin{array}{l}\text { Mediadores profissionais de } \\
\text { qualidade e segurança do paciente } \\
\text { como estratégia para cuidados de } \\
\text { saúde seguros. }\end{array}$ & $\begin{array}{l}\text { Como forma de reduzir os erros na assistência } \\
\text { hospitalar relacionados a segurança do paciente, } \\
\text { é necessário que haja comunicação e } \\
\text { gerenciamento entre a equipe multiprofissional } \\
\text { do cliente. }\end{array}$ \\
\hline $\begin{array}{l}\text { BOHRER et al., } \\
2016 .\end{array}$ & $\begin{array}{l}\text { Comunicação e Cultura de Segurança } \\
\text { do Paciente no Ambiente Hospitalar: } \\
\text { Visão da Equipe Multiprofissional. }\end{array}$ & $\begin{array}{l}\text { A colaboração e comunicação das equipes na } \\
\text { rede hospitalar são barreiras que impedem o } \\
\text { atendimento seguro e a promoção da segurança } \\
\text { do paciente. }\end{array}$ \\
\hline Autores & Título & Síntese/considerações \\
\hline $\begin{array}{l}\text { WAMI et al., } \\
2016 .\end{array}$ & $\begin{array}{l}\text { Cultura de segurança do paciente e } \\
\text { fatores associados: um estudo } \\
\text { quantitativo e qualitativo da visão dos } \\
\text { profissionais de saúde nos hospitais } \\
\text { Jimma Zone, sudoeste da Etiópia. }\end{array}$ & $\begin{array}{l}\text { Para a evolução no processo de } \\
\text { assistência ao paciente é necessário que se tenha } \\
\text { cooperação, abertura para a comunicação e troca } \\
\text { de ideias entre os profissionais de saúde. }\end{array}$ \\
\hline $\begin{array}{l}\text { TARTAGLIA et } \\
\text { al., } 2018 .\end{array}$ & $\begin{array}{l}\text { Comunicação,comportamentos } \\
\text { destrutivos e segurança do paciente. }\end{array}$ & $\begin{array}{l}\text { Se faz necessário a conscientização das } \\
\text { organizações de saúde sobre a problemática que } \\
\text { a falta de comunicação traz a segurança do } \\
\text { paciente, esse comportamento em um ambiente } \\
\text { tão sério é intolerável se for considerar os riscos } \\
\text { que estão em jogo. }\end{array}$ \\
\hline $\begin{array}{l}\text { DINIUS et al., } \\
2019 .\end{array}$ & $\begin{array}{l}\text { Pilotar e avaliar a viabilidade de um } \\
\text { programa de treinamento para } \\
\text { melhorar a segurança do paciente para } \\
\text { equipes interprofissionais de } \\
\text { internação - Protocolo de estudo de } \\
\text { um ensaio clínico controlado } \\
\text { randomizado por agrupamento }\end{array}$ & $\begin{array}{l}\text { A comunicação entre diferentes profissionais, } \\
\text { além de contribuir para o espessamento de } \\
\text { conhecimento, atende as necessidades de } \\
\text { segurança do paciente. Contribui também para o } \\
\text { atendimento padronizado de competências } \\
\text { básicas no serviço de saúde. }\end{array}$ \\
\hline $\begin{array}{l}\text { HEMESATH et } \\
\text { al., } 2019 .\end{array}$ & $\begin{array}{lcr}\text { Comunicação } & \text { Eficaz } & \text { nas } \\
\text { Transferências } & \text { Temporárias } & \text { do } \\
\text { Cuidado de Pacientes Hospitalizados. }\end{array}$ & $\begin{array}{l}\text { Comunicação eficaz depende de uma } \\
\text { padronização nos serviços de saúde, como a } \\
\text { elaboração de formulários e informações que } \\
\text { assegurarão as informações de segurança do } \\
\text { paciente, disponível para as equipes de saúde. }\end{array}$ \\
\hline Autores & Título & Síntese/considerações \\
\hline $\begin{array}{l}\text { BIASIBETTI et } \\
\text { al., } 2019 .\end{array}$ & $\begin{array}{l}\text { Comunicação Para a Segurança do } \\
\text { Paciente em Internações Pediátricas. }\end{array}$ & $\begin{array}{l}\text { A comunicação entre as equipes de saúde possui } \\
\text { lacunas e estratégias precisam ser elaboradas } \\
\text { para que a assistência ao paciente venha a ser } \\
\text { qualificada, integralizada e segura. }\end{array}$ \\
\hline $\begin{array}{l}\text { SANTOS et al., } \\
2018 .\end{array}$ & $\begin{array}{l}\text { Comunicação no Handoff na Terapia } \\
\text { Intensiva: Nexos com a Segurança do } \\
\text { Paciente. }\end{array}$ & $\begin{array}{l}\text { É necessário que haja a manutenção de um } \\
\text { documento padronizado para o preenchimento, } \\
\text { dessa forma, haverá otimização do tempo, } \\
\text { melhor desempenho na assistência ao paciente e } \\
\text { uma comunicação mais efetiva. }\end{array}$ \\
\hline
\end{tabular}

Fonte: Dados da pesquisa, 2021. 


\section{Discussão}

A promoção da segurança do paciente tem sido bastante desafiadora durante o século XXI, pois está atrelada aos cuidados multiprofissionais em saúde. Dessa forma, a temática tem sido geradora de discussões nacionais e internacionais, pelo fato de haver divulgações a respeito do assunto entre profissionais e pacientes. Além disso, as diferentes abordagens sobre o assunto foram desmistificadas, pois a Organização Mundial de Saúde (OMS) criou planos estratégicos para que as instituições de saúde pudessem utilizar, a fim de prevenir agravos e evitar com que os erros venham a acontecer (OLINO et al., 2019).

Desse modo, os órgãos públicos, ainda preocupados com essa problemática recorrente, desenvolveram, em 2004, uma Aliança Mundial para a Segurança do Paciente cujo objetivo era desenvolver práticas políticas que abrangessem diversos países, em busca de melhorias nos grandes problemas assistenciais, por meio da elaboração de metas internacionais, destacandose a qualidade na comunicação efetiva (OLINO et al., 2019).

A comunicação efetiva, quando implementada na ótica da equipe multiprofissional de saúde precisa ocorrer de maneira clara, direta, conjunta e rotineira (BIASIBETTI et al., 2019). No ambiente hospitalar, os pacientes são encaminhados para inúmeros setores, onde passam por diversos profissionais e procedimentos, com isso, a comunicação intersetorial torna-se imperativa para uma assistência de qualidade, conforme mencionado por Hamesath et al. (2019). Sendo assim, essa interação tem sido amplamente estimulada pelas autoridades internacionais, a exemplo da OMS, como uma ferramenta indispensável para a garantia da segurança do paciente (LEMOS et al., 2019).

Segundo o estudo realizado por Nogueira e Rodrigues (2015), o processo de interatividade da equipe multiprofissional reflete diretamente na prestação de cuidados, bem como na segurança do paciente, uma vez que falhas durante esse processo podem corroborar erros médicos. Desse modo, quando ocorre uma ineficiência ao longo da comunicação, os efeitos são notórios, resultando em danos que podem vir a comprometer a qualidade de vida do cliente (BIASIBETTI et al., 2019; CHILDRESS, 2015; LEMOS et al., 2019).

No tocante ao processo de comunicação efetiva, tem sido uma prática bastante complexa, pois requer uma grande interação social que demanda da participação ativa tanto dos profissionais envolvidos, quanto dos gestores e pacientes. De acordo com estudos brasileiros, os profissionais de saúde atuantes em hospitais da rede pública enfrentam os mais diversos conflitos e divergências entre as categorias, desse modo, isso acaba por tornar cada vez mais 
profissionais desunidos e incapazes de prestar apoio um ao outro, sem o efetivo trabalho em equipe e engajamento em prol de um único propósito, nesse caso, dificilmente será garantida a segurança do paciente (TARTAGLIA et al., 2018).

Dessa forma, a falta de comunicação além de provocar grandes impactos à vida do paciente, traz consequências no campo profissional e pessoal dos profissionais de saúde, esse tipo de comportamento torna-se uma ameaça por afetar diretamente a qualidade do trabalho em equipe, quebra dos laços de confiança causando uma verdadeira desarmonia ao ambiente de trabalho (MOREIRA et al., 2019).

No que tange as dificuldades para a efetivação dessa atuação conjunta entre os profissionais de saúde, é possível destacar ainda que transições de plantão inadequadas, falta de estímulo pela gestão hospitalar e a ideia de hierarquia entre as profissões configuram-se como as principais causas do problema (HAMESATH et al., 2019; NOGUEIRA; RODRIGUES, 2015). Nesse sentido, a colaboração de todos nesse processo é primordial, uma vez que a assistência depende tanto da equipe multiprofissional, quanto da gestão hospitalar, que sistematiza e ordena a prestação de serviços (FASSARELA et al., 2017).

A passagem de plantão, ferramenta fundamental na comunicação entre os profissionais de saúde e na compreensão do quadro clínico do cliente, é um aspecto de suma importância que necessita ser melhor aprimorado (CHILDRESS, 2015). Sendo assim, mediante o exercício adequado da transição de plantão, a assistência torna-se continuada e padronizada, porém essa troca de informações no ambiente hospitalar muitas vezes não ocorre de maneira satisfatória entre as diferentes categorias profissionais (CORPOLATO et al., 2019; BOHER et al., 2016). As longas jornadas de trabalho e as elevadas demandas encontradas por esses profissionais levam a ineficiência no processo de comunicação efetiva.

Igualmente, outro problema que dificulta a implementação de uma comunicação efetiva pela equipe multidisciplinar é a hierarquia existente entre as profissões. Pois, ainda nos dias atuais é comum haver barreiras que impedem a comunicação fluida entre profissionais de áreas distintas (LEMOS et al., 2019). Nogueira e Rodrigues (2015) afirmam em seu estudo que profissionais que exercem a mesma profissão tendem a se comunicarem melhor, enquanto que Biasibetti (2019) mostra pesquisas que apontam a hierarquia, ainda evidente entre as profissões, como um fator que pode inibir o diálogo entre os colegas de trabalho.

Diante do estudo realizado por Freire et al. (2019), os hospitais brasileiros preocupados com a insatisfação que vem sendo demonstrada pelos usuários dos serviços de saúde, estão aderindo ao sistema de acreditação, que é operado pala Organização Nacional de Acreditação 
(ONA), onde são estabelecidos padrões requeridos nas instituições, a fim de receberem avaliações e certificações pela comprovação da qualidade dos serviços de saúde ofertados. Foi pensando nas ocorrências de erros e agravos a saúde dos pacientes bem como na minimização dos mesmos que resolveram aderir aos programas de acreditação de qualidade no âmbito hospitalar, tais estratégias têm demonstrado melhorias tanto a comunicação oral com na escrita, além de diminuir os custos com gastos na assistência visa desenvolver o papel de consciência e mudança no comportamento dos colaboradores com o intuito de melhorar a qualidade da prestação dos serviços.

Mediante o tema em questão e conforme estudos realizados em Hospitais Universitários Públicos no Brasil, a exemplo de Guzinski et al. (2019), tem se estimulado as discussões acerca da importância em garantir a segurança do paciente, tal situação vem mostrando inquietação dos gestores atuantes nessas unidades. Por isso, tem se tornado um desafio tanto para eles como para os profissionais de saúde em abolir culturas e hábitos antigos, para aderir e se adequar as novas mudanças de atitudes a fim de beneficiar as boas práticas em sua vivência assistencial.

Percebe-se, que são marcantes os avanços tecnológicos na tentativa de estimular a comunicação efetiva, apesar das vantagens dessas ferramentas nenhum meio de comunicação tem se mostrando tão eficaz como a comunicação verbal, porque possibilita que haja a troca de informações precisas sobre os pacientes, o referido estudo destaca ainda que quando os cuidados seguem as condutas adequadas há a diminuição tanto nos riscos como nos possíveis danos que podem vir acometer a saúde do paciente o que corrobora para favorecer que a efetiva prática seja realizada com segurança.

\section{Conclusão}

A assistência ao paciente está bastante atrelada à equipe multiprofissional, dado isso sabe-se da necessidade de uma comunicação efetiva entre os profissionais que compõem essas equipes, de modo que seja possível reduzir danos para o paciente e melhorar sua segurança, consequentemente, havendo um aumento dos desfechos clínicos favoráveis. Para que isso ocorra, torna-se necessário que a comunicação seja clara, direta e rotineira entre os profissionais. Desse modo, a interatividade entre a equipe, além de melhorar a segurança e o cuidado do paciente, interfere na prestação dos cuidados e no bem-estar dos profissionais, uma 
vez que a falha da comunicação pode tanto causar estresse entre eles quanto corroborar para erros durante a assistência.

\section{Referências}

BIASIBETTI, C. et al. Comunicação para a segurança do paciente em internações pediátricas. Revista Gaúcha de Enfermagem, v. 40, p.1-9, 2019.

BOHRER, C.D. et al. Comunicação e Cultura de Segurança do Paciente no Ambiente Hospitalar: Visão da Equipe Multiprofissional. Revista de Enfermagem da UFSM, v. 6, n.1, p.50-60, 2016.

BRASIL. Ministério de Saúde. Portaria n 2.095, 24 de setembro de 2013. Aprova Protocolos Básicos de Segurança, 2013.

CHILDRESS, S.B. Team Communication: It's About Patient Safety. J Oncol Pract. v. 11, n.1, p.23-25, 2015.

CORPOLATO, R.C. et al. Padronização da passagem de plantão em Unidade de Terapia Intensiva Geral Adulto. Revista Brasileira de Enfermagem, v. 72, n.1, p.95-102, 2019.

DINIUS, J. et al. Piloting and evaluating feasibility of a training program to improve patient safety for inter-professional inpatient care teams - study protocolo of a cluster randomized controlled trial. Trials, v. 20, p.1-11, 2019.

FASSARELLA, C.S. et al. Profissionais Mediadores da Qualidade e Segurança do Paciente como Estratégia para o Cuidado Seguro. Revista Mineira de Enfermagem, v. 21, p.1-7, 2017.

FREIRE, E.M.R. et al. A comunicação como estratégia para manutenção da acreditação hospitalar. Escola Anna Nery, v. 23, n.1, p.1-9, 2019.

GUZINSKI, C. et al. Boas práticas para comunicação efetiva: A experiência do round interdisciplinar em cirurgia ortopédica. Rev. Gaúcha Enferm., v.40, p.1-5, 2019.

HEMESATH, M.P. et al. Comunicação eficaz nas transferências temporárias do cuidado de pacientes hospitalizados. Revista Gaúcha de Enfermagem, v. 40, p.1-6, 2019.

Instituto Brasileiro para Segurança do Paciente. Comunicação ineficaz está entre as causasraízes de mais de 70\% dos erros na atenção à saúde. IBSP, 2017.

LEMOS, D.M.P. et al. Comunicação efetiva para o cuidado seguro ao paciente com implante de dispositivo de assistência ventricular. Revista Gaúcha de Enfermagem, v.40, p.1-5, 2019.

MOREIRA, F. T. L. S. et al. Estratégias de comunicação efetiva no gerenciamento de comportamentos destrutivos e promoção da segurança do paciente. Rev. Gaúcha Enferm, v. 40, p.1-9, 2019. 
NOGUEIRA, J. W. S. N., RODRIGUES, M.C.S. Comunicação Efetiva no Trabalho em Equipe em Saúde: Desafio para a Segurança do Paciente. Cogitare Enfermagem, v. 20, n.3, p.636640, 2015.

OLINO, L. et al. Comunicação efetiva para a segurança do paciente: nota de transferência e Modified Early Warning Score. Rev Gaúcha Enferm, n.40, p.1-9, 2019.

PEREIRA, F.G.F. et al. SEGURANÇA DO PACIENTE E PROMOÇÃO DA SAÚDE: uma reflexão emergente. Revista Baiana de Enfermagem, v. 23, n.1, p.1-9, 29(3):271-278, 2015.

SANTOS, D. et al. Segurança do paciente: uma abordagem acerca da atuação da equipe de enfermagem na unidade hospitalar. Revista Temas em Saúde, v. 17, n.2, p.213-225, 2017.

SANTOS, G.R.S. et al. Comunicação no handoff na terapia intensiva: nexos com a segurança do paciente. Escola Anna Nery, v. 22, n.2, p.1-12, 2018.

SIMAN, A.G. et al. A prática de notificação de eventos adversos em um hospital de ensino. Rev Esc Enferm USP, 51e03243, 2017.

TARTAGLIA, A. et al. Communication, destructive behaviors and patient safety. Rev. Sobecc, v. 23, n.4, p.226-230, 2018.

WAMI, S.D. et al. Patient Safety Culture And Associated Factors: A Quantitative And Qualitative Study Of Healthcare workers' View In Jimma Zone Hospitals, Southwest Ethiopia. BMC Health Services Research, v.16, p. 495-505, 2016.

\section{Como citar este artigo (Formato ABNT):}

SANTOS, Tatiane de Oliveira; LIMA, Maria Adriely Cunha; ALVES, Victória Santos; RIBEIRO, Maria Caroline Andrade ; ALVES, Raquel Santos ; SOUZA, Mércia Rocha; CORREIA, Fernanda Vasconcelos Prado; OLIVEIRA, Ana Carolina Amorim; SALES, Larissa Ferreira; OLIVEIRA, Halley Ferraro. Comunicação efetiva da equipe multiprofissional na promoção da segurança do paciente em ambiente hospitalar. Id on Line Rev.Mult. Psic., Maio/2021, vol.15, n.55, p. 159-168. ISSN: 19811179 .

Recebido: 14/03/2021;

Aceito: 18/03/2021. 